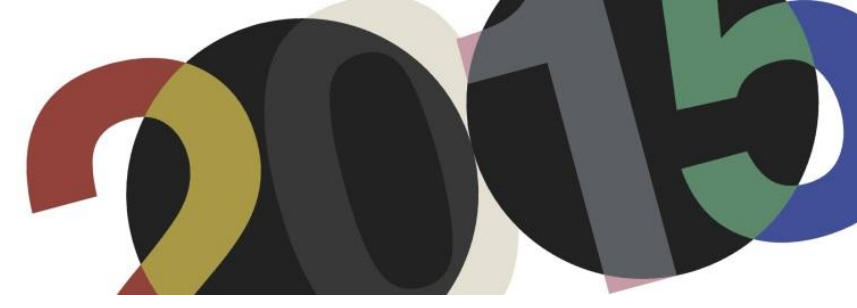

DOI: http://dx.doi.org/10.4995/LC2015.2015.570

\title{
Criterios de combinación de colores para la arquitectura en Salubra I: estudio de tonos
}

\author{
J. Serra, J. Llopis, A. Torres y M. Giménez
}

Escuela Superior de Arquitectura de Valencia

Resumen: Realizamos un nuevo estudio de las combinaciones de colores seleccionados por Le Corbusier en los 'claviers' para la empresa de papeles pintados Salubra en 1931, mediante su análisis en el espacio de color Natural Color System NCS, que permite comprender las variables perceptivas de los colores (tono, negrura y cromaticidad) y sus criterios de armonía.

En este artículo discutimos las gamas tonales seleccionadas y aquellas que están ausentes., e intentamos desentrañar el orden subyacente en las combinaciones de colores mediante el estudio de las similitudes y contrastes de sus tonalidades en NCS. De manera gráfica, se demuestran algunos principios de armonía como el recurso habitual de contrastar tonos fríos con cálidos, algo que difiere ligeramente del tradicional contraste de complementariedad. El trabajo completo ha sido publicado en Color Research and Application () Wiley Periodicals. Color combination criteria in Le Corbusier's Purist architecture based on Salubra claviers from 1931. DOI: 10.1002/col.21940.

Abstract: We have carried out a new study of the color combinations selected by Le Corbusier in the 'claviers' for the Salubra wallpaper company in 1931, by analyzing them in the Natural Color System NCS, which allows us to understand the perceptive variables of colors (hue, blackness and chromaticness) as well as their combination criteria.

In this paper, the selected hue ranges are discussed, and those absent ones. We aim to find out the underlying order in the color combinations by studying the similarities and contrasts of their hues in NCS. We demonstrate graphically some principles in Le Corbusier's color preferences, such as the usual resource of contrasting cool with warm colors, something slightly different to the traditional contrast of complementary colors. The complete work has been published in Color Research and Application () Wiley Periodicals. Color combination criteria in Le Corbusier's Purist architecture based on Salubra claviers from 1931. DOI: 10.1002/col.21940.

Palabras clave: color, armonía; Le Corbusier; Salubra; arquitectura; Natural Color System. Keywords: color; harmony; Le Corbusier; Salubra; architecture; Natural Color System.

\section{Introducción}

En 1931 la empresa de papeles pintados Salubra, con sede en Basilea (Suiza), encarga a Le Corbusier confeccionar una primera carta de colores para la arquitectura que se completa en 1959 con una segunda versión. La primera de estas colecciones estaba pensada como una serie armónica, de manera similar a como se organizan los sonidos en un piano, de ahí su denominación como "claviers de couleurs" (fig. 1). En 1997, ambas colecciones de colores se reeditan junto con el texto inédito de Le Corbusier Polychromie Architecturale fechado entre finales de 1932 y principios de $1933^{1}$, sin duda el documento más importante para comprender el color en la obra del arquitecto suizo.

\footnotetext{
${ }^{1}$ Le Corbusier: "Polychromie architecturale". En Rüegg, Arthur (Ed.): Polychromie architecturale: Le corbusier's color keyboards from 1931 and 1959. Basel: Birkhäuser, 1997. pp. 94-143.
} 
Son varios los autores que han estudiado detenidamente las cartas de color Salubra I: JL. Caivano explica su organización y funcionamiento ${ }^{2}$; M. Colli la ordenación de los colores en los claviers $^{3}$; A. Ruegg analiza en profundidad las cartas junto con su texto Polychromie Architecturale $e^{4}$ y J. de Heer ${ }^{5}$ desarrolla un estudio exhaustivo de los aspectos cromáticos en los escritos y la arquitectura Purista de Le Corbusier, evidenciando el alto grado de libertad con el que finalmente disponía los colores en la obra construida más allá de los claviers de color Salubra I.

Sin embargo, existe un asunto que no se ha trabajado hasta la fecha, que consiste en un análisis de las combinaciones de colores seleccionados en los teclados empleando un espacio de notación del color estándar como Natural Color System (NCS), que permite comprender las características colorimétricas de los colores (tono, negrura y cromaticidad) y sus criterios de armonía. En este artículo nos centramos en el estudio de los tonos de los colores, aunque también se han estudiado la negrura y la cromaticidad ${ }^{6}$.

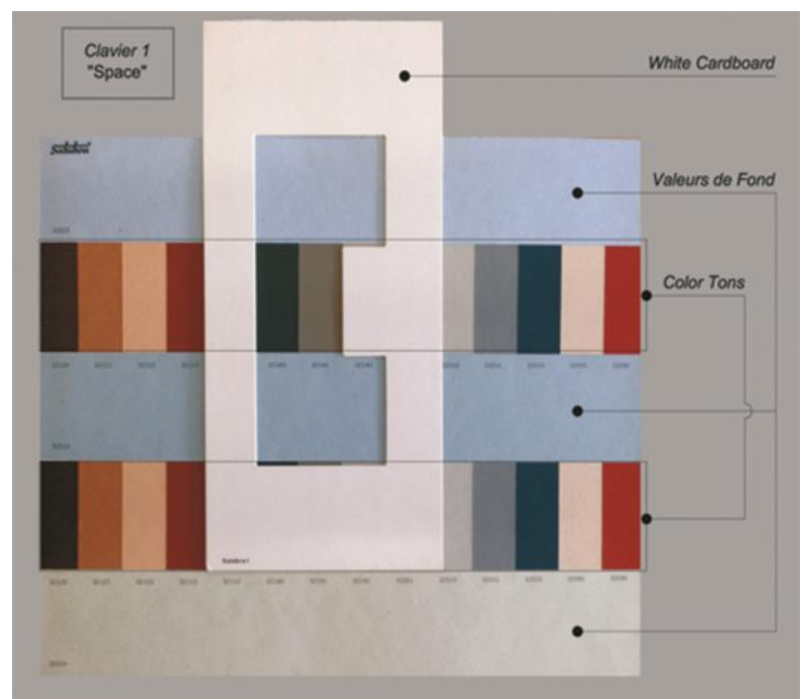

1. Foto del clavier 'Espace' de la colección Salubra I (1931), indicando los valeurs de fond y los color tons, así como el cartón blanco para ayudar a seleccionar cada combinación.

Este análisis es coherente con el pensamiento de Le Corbusier, al menos en su etapa Purista a la que pertenece esta colección de colores, quien consideraba que la belleza en la arquitectura respondía a una serie de leyes basadas en los números que podían ser conocidas y estudiadas ${ }^{7}$. En este sentido, su aproximación a la belleza

\footnotetext{
${ }^{2}$ Caivano, José Luis: La compleja relación con el color de uno de los maestros de la arquitectura moderna: Nueva edición de la Policromía Arquitectónica de Le Corbusier”. En Luminotecnia. N N 84. Mar de la Plata (Argentina): Editores SRL, 2007. pp. 58-61.

${ }^{3}$ Colli, Luisa Martina: “Hacia una policromía arquitectónica”. En Lucan, Jaques (Ed.): Le Corbusier, une encyclopédie. Paris: Centre de Crêation Industrielle, 1987. pp. 97-100.

${ }^{4}$ Rüegg, Arthur: "Le Corbusier's Polychromie architecturale and his Color keyboards from 1931 and 1959". En Rüegg, Arthur (Ed.): Polychromie architecturale: Le Corbusier's color keyboards from 1931 and 1959. Basel: Birkhäuser, 1997. pp. 12-93.

${ }^{5}$ Heer, Jean de: The architectonic colour: polychromy in the Purist architecture of Le Corbusier. Rotterdam: 010 Publishers, 2009.

${ }^{6}$ Serra Lluch, Juan y otros: "Color combination criteria in Le Corbusier's purist architecture based on Salubra claviers from 1931”. En: Color Research and Application. Enero 2015 (Early view). Wiley Periodicals, 2015. DOI: 10.1002/col.21940

${ }^{7}$ Dempsey, Amy: Estilos, escuelas y movimientos: guía enciclopédica del arte moderno. Barcelona: Blume, 2002.
} 
ideal responde a lo que podemos considerar un paradigma positivista ${ }^{8}$ y se fundamenta en criterios racionales de orden y proporción. No obstante, Le Corbusier no concibe las cartas Salubra I como un sistema abierto que sirviera para combinar cualquier color de la naturaleza, sino como una especie de manual muy bien delimitado de sólo 43 colores que tienen todas las garantías de ser adecuados para la arquitectura y establece cómo combinarlos. Le Corbusier no busca un sistema de clasificación de todos los colores posibles, sino más bien un instrumento que permita restringirlos y que garantice las posibilidades de armonía. De ahí que las cartas de color se construyan al modo del teclado de un piano, en el que las teclas son limitadas pero suponen la base de cualquier armonía musical.

En todo caso, Le Corbusier limita el margen de libertad según un criterio de armonía absolutamente personal, que sólo puede ser explicado desde su sensibilidad como arquitecto y como pintor, así como desde su deseo de racionalizar la mayor parte de sus procesos creativos.

\section{Método}

El estudio se realiza partiendo de la documentación disponible en la Fundación Le Corbusier en París, donde las cartas de color originales se han evaluado visualmente y con instrumental técnico (espectrofotómetro de contacto modelo Kónica Minolta CM 700d, colorímetro Munsell y NCS) ${ }^{9}$.

Nos centramos en la carta Salubra I de 1931 porque responde a inquietudes que Jeanneret y Ozenfant empezaron a investigar seis años antes en su texto Nature et création $^{10}$, donde consideraban que la belleza se basaba en el orden y la claridad formal.

Nuestra metodología ha consistido en extraer todas las combinaciones posibles de colores de la colección, que resultan ser 312 combinaciones de 4 colores (1.248 parejas de colores) y representarlas en el espacio de color tridimensional NCS, que tiene forma de doble cono invertido. Esta representación es un modelo virtual navegable que permite ser observado desde cualquier punto de vista (fig. 2), identificando si existen criterios de armonía o contraste, o sea coincidencias o divergencias con respecto a los tonos de los colores o a sus valores de negrura y cromaticidad.

\footnotetext{
${ }^{8}$ O'Connor, Zenna: “Colour Harmony Revisited”. En: Color Research and Application. № 35 (4). Wiley Periodicals, 2009. pp. 268-273.

${ }^{9}$ En los casos en los que el paso del tiempo había deteriorado el color de las muestras, como en el caso del azul 32040, se extrajeron las características colorimétricas de las cartas publicadas en 1997, pues se considera que son una buena reinterpretación de los colores originales.

${ }^{10}$ Jeanneret, Ozenfant: "Nature et creation”. En: L'Esprit Nouveau. N 19. Paris: Ed. de L’Esprit Nouveau, 1923. s.p.
} 


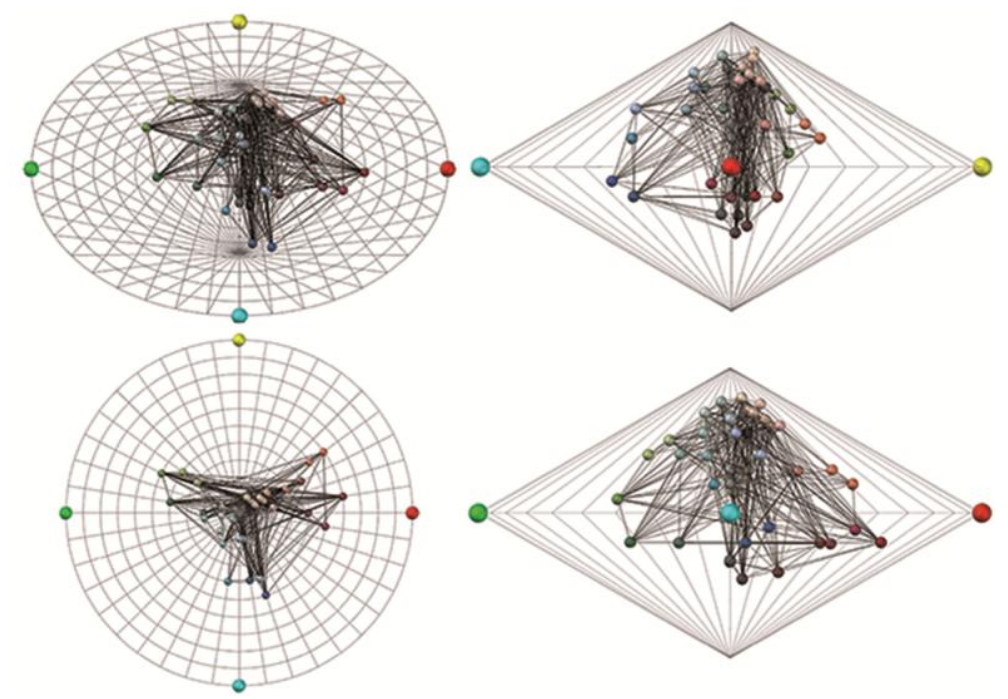

2. Modelo virtual navegable en 3D con indicación de todas las parejas de colores Salubra I (1931) en el espacio de color NCS. Las líneas que unen las esferas indican colores emparejados.

A nivel teórico, la búsqueda de los criterios de combinación de los colores se ha llevado a cabo siguiendo los modelos habituales del paradigma positivista, que son los más cercanos al pensamiento de Le Corbusier. Por un lado la búsqueda de similitudes entre los colores, como proponía A. Munsell ${ }^{11}$ o posteriormente J. Itten ${ }^{12}$; por otro la búsqueda de equilibrios entre fuerzas oponentes, como sostenía el propio A. Munsell, Chevreul ${ }^{13}$ ó Goethe $^{14}$, entre otros. Es cierto que todos los modelos de agrupación de colores basados en similitudes y contrastes son limitados, sobre todo cuando aspiran a dar respuestas estéticas universales, válidas para cualquier contexto o cualquier observador, como advierte muy bien la arquitecta Z. O'Connor ${ }^{15}$. La intención de Le Corbusier no era la de predecir la respuesta humana ante sus combinaciones de color sino establecer su propio criterio de agrupación, basado en sus preferencias personales. En este sentido, nuestro objetivo es comprender mejor unas combinaciones de color que el propio Le Corbusier ya ha considerado apropiadas para la arquitectura. En todo caso, es difícil caer en el error de considerar las cartas Salubra I como la solución universal al problema de las armonías de color, cuando ni siquiera el propio Le Corbusier parece que las empleara de manera estricta.

\section{Análisis de los tonos NCS}

Los 43 colores de la colección Salubra I se organizan en 12 colecciones denominadas claviers. Cada una de ellas consta de tres colores de fondo (valeurs de fond) y dos bandas horizontales de color tons colocados en una secuencia que asemeja a un teclado. Para facilitar la selección de los colores, las cartas se acompañan de unos cartones de color blanco que permiten aislar dos valeurs de fond junto con uno o dos color tons, de modo que los

\footnotetext{
${ }^{11}$ Munsell, Albert: A grammar of colour. Mittineague, Massachusetts: The Strathmore paper company, 1921.

${ }^{12}$ Itten, Johannes: Kunst der Farbe. Ravensburg: Otto Maier Verlag, 1961. Versión inglesa por: Hagen, E. The elements of color. NY: Van Nostrand Reinhold, 1970.

${ }^{13}$ Chevreul, Michel Eugène: De la loi du contraste simultané des couleurs et de l'assortiment des objets colorés. Paris: Gauthier-Villars et fills; 1839. p 750. Traducción inglesa: Martel, C: The principles of harmony and contrast of colors and their applications to the arts. London: Longman, Brown, Green and Longmans, 1855.

${ }^{14}$ Goethe, Johann Wolfgang: Materialien zur Geschichte der Farbenlehre, Zur Farbenlehre. Tubingen: Cotta, 1810. Traducción inglesa: Eastlake, C.: Goethe's theory of colours. London: J. Murray, 1840.

${ }^{15}$ O’Connor. Op. cit., p. 268.
} 
colores seleccionados, con un máximo de cuatro, deben ser observados aislados con un cartón blanco. En las distintas cartas de color parece evidente que los valeur de fond son los que dan el "timbre" a cada clavier y se asocian de algún modo con los nombres propios que los identifican: Espace, Ciel, Velour I y II, Sable I y II, Mur I y II, Paysage y Bigarrées I, II y III. Mediante un "proceso de asociación”, los valeurs de fond se vinculan a un concepto, a una idea de orden secundario. Así, a los colores azules corresponde la idea asociada de "cielo" y "espacio", a los ocres la "arena", a los rojos la "pared" y a los verdes el "paisaje". Este papel asociativo del color es evidente en proyectos como las viviendas en Pèssac, donde el verde se asocia a la idea de bosque lejano o de cubierta ajardinada, el azul al fondo del cielo, y el rojo se emplea para "fijar" una fachada como "pared" y reforzar su presencia.

Los 43 colores en la colección Salubra I derivan de 14 colores iniciales a los que denominamos colores principales. A partir de cada uno de estos colores principales, Le Corbusier obtuvo varias familias de colores a base de añadir pigmento blanco y así desplazar los colores principales a una versión más suave en tres o cuatro pasos. Así pues, encontramos grupos de colores que se originan a partir de un mismo color principal y diremos que pertenecen a la misma familia de color-pigmento (azules cobalto o rojos bermellón, por ejemplo). Cada uno de los colores de la colección original Salubra I se denotaba con un número de tres dígitos, correspondiendo a los colores principales los siguientes: 001, 010, 020, 030, 040, 050, 060, 080, 090, 100, 110, 120, 130 y 140. Las unidades de cada número de referencia indican aproximadamente la negrura y cromaticidad del color, mientras que las decenas y centenas identifican cada una de las 14 familias de color-pigmento. Siguiendo la denominación de M. colli para cada familia de color-pigmento: $\approx$ blanco $^{16}$ (primeros dos dígitos 00: 001), grises (001, 010, 011, 013), azules ultramar (primeros dos dígitos 02: 021, 022, 023, 024), azules cobalto (primeros dos dígitos 03: 030, 031, 032, 033, 034), verdes veronés (primeros dos dígitos 04: 040, 041, 042), verdes amarillentos (primeros dos dígitos 05: 050, 051, 052, 053), naranjas (primeros dos dígitos 08: 080, 081, 081), rojos bermellón (primeros dos dígitos 09: 090, 091), carmines (primeros dos dígitos 10: 100, 101, 102), ocres rojizos (primeros dos dígitos 11: 110, 111, 112); sienas (primeros dos dígitos 12: 120, 121, 122, 123), marrones (primeros dos dígitos 13: 130, 131) y sombras (primeros dos dígitos 14: 140, 141, 142, 150).

\footnotetext{
${ }^{16}$ Colli llama a este primer color "blanco", aunque no pertenece estrictamente a los colores neutros en NCS (S0510 Y30R). Para evitar confusiones, denominamos a esta familia de color-pigmento con un solo miembro " $\approx$ blanco".
} 


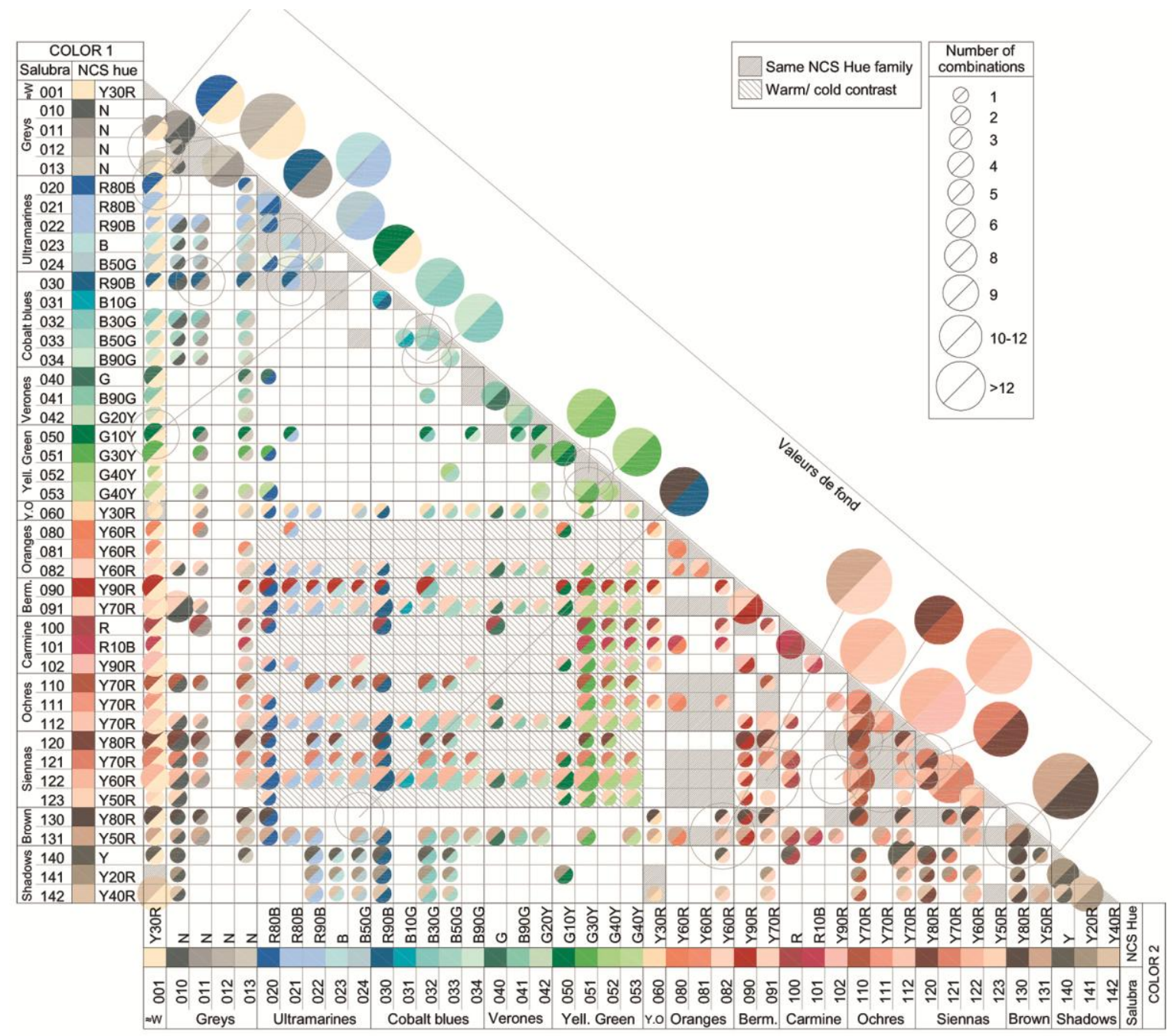

3. Representación de todas las parejas de colores Salubra I (1931). En abscisas y ordenadas se muestran los 43 colores con su notación NCS. El número de veces que se repite una pareja en los claviers se indica con el tamaño del círculo. Aquellas parejas correspondientes a dos colores de fondo (valeurs de fond) se representan al margen, por ser mucho más numerosas. El tramado denso de rayas indica el lugar geométrico de las combinaciones de colores de igual tono en NCS. El tramado de rayas menos denso indica el lugar geométrico de las combinaciones de colores con contraste frío/ cálido ${ }^{17 .}$

Al seleccionar estas 14 familias de color-pigmento y no cualesquiera otras, Le Corbusier quiere evitar aquellos colores que no sean plenamente "arquitectónicos" y que por lo tanto puedan neutralizar el efecto visual de la forma arquitectónica. En el ámbito de la pintura de caballete, Ozenfant y Jeanneret daban prioridad a los volúmenes y el dibujo de las formas frente al color y deseaban evitar el carácter destructivo del color que criticaban en otros movimientos artísticos como De Stijl. También en la arquitectura debía evitarse todo efecto impresionista del color que pudiera distorsionar la geometría de las formas puras.

\footnotetext{
${ }^{17}$ Téngase en cuenta que el patrón de color impreso puede haber sufrido modificaciones respecto al color verdadero a causa del proceso de impresión. Refiérase siempre a cartas originales NCS.
} 


\subsection{Colores presentes}

En el texto Le Purisme ${ }^{18}$, previo a la primera colección de colores Salubra I, Ozenfant y Jeanneret describían tres gamas de color para la pintura de caballete. La primera de ellas era la grande gamme que contenía todos aquellos colores 'constructivos' que fueron empleados por los grandes pintores de la historia del arte desde Miguel Ángel o Ingres, pasando por Seurat o Cezanne, hasta los cubistas Picasso o Gris. Son pintores bien considerados porque todos ellos, en opinión de Le Corbusier, subordinaron el color al dibujo y dieron prioridad a la construcción de la forma mediante el claroscuro. Optaron por el disegno frente al colore, siguiendo la oposición entre estos dos conceptos que se inicia en época Renacentista con Vasari y se extiende hasta finales del s. XIX en Francia. La grande gamme incluye el amarillo, ocre rojizo, colores tierras, blanco, negro ultramar y los colores derivados de ellas. La segunda es una gamme dynamique y a diferencia de la anterior contiene colores que no son "estáticos", sino que dan la sensación de continuo cambio de plano. A ella pertenecen el amarillo limón, los naranjas, el rojo bermellón, verde veronés o el azul cobalto claro. La tercera es una gamme de transition que contiene al verde esmeralda y los colores esmaltados, sin cualidades constructivas. Le Corbusier da a entender que sólo desea emplear la grande gamme.

En el texto Polychromie Architecturale, el criterio para seleccionar los colores también responde a un deseo de restringirlos a sólo aquellos que sean "verdaderamente arquitectónicos", la selección de un gamme noble ${ }^{20}$ : blanco, negro, ultramar, azul, verde inglés, ocre amarillo, tierra siena natural, bermellón, carmín, rojo inglés y tierra siena tostada. Se abandona por lo tanto la distinción inicial entre tres gamas de color y se observa que para Salubra I Le Corbusier suma sin dificultad a la grande gamme algunos colores que pertenecen a la gamme dynamique, la "gama ágil e inquieta que da la sensación de continuo cambio, 21 .

Si observamos los 43 colores seleccionados en Salubra I vemos que aproximadamente la mitad de ellos (17) pertenecen a familias comprendidas entre NCS R10B y Y80R, o sea colores fríos, y la otra mitad (17) pertenece a familias ubicadas entre NCS G40Y y R80B, o sea colores cálidos. Quedarían al margen los colores grises y sombras (9). Debe señalarse que las gamas de colores cálidos incluyen sólo seis tonos NCS organizados alrededor del rojo Y80R mientras que las gamas frías contienen el doble de tonos NCS, seis alrededor del azul R80B y seis alrededor del verde G30Y. Por lo tanto los colores cálidos que están excesivamente próximos al amarillo o al violeta se evitan. Como discutiremos más adelante la oposición entre tonos fríos y cálidos fue importante en el pensamiento de Le Corbusier.

Además las gamas cálidas que Le Corbusier elige son mayoritariamente marrones y grises, es decir colores terciarios que se corresponden con porcentajes pequeños de cromaticidad en NCS. Estos colores pueden ser explicados por el deseo de Le Corbusier de conectarlos con los pigmentos naturales. Para Le Corbusier son estos colores derivados de la tierra los que han acompañado a la arquitectura desde sus orígenes. Son colores "eternos" que se distinguen de los pigmentos sintetizados químicamente, tan populares en la pintura de la segunda mitad del siglo XIX y que Le Corbusier consideraba demasiado saturados y no arquitectónicos. Aun así las investigaciones recientes de los centros históricos de muchas de las ciudades, al menos en el arco mediterráneo,

\footnotetext{
${ }^{18}$ Jeanneret, Ozenfant. Op. cit., p. 382 ff.

19 'éminemment architecturales', Le Corbusier, Polychromie Architecturale, Op. cit. p 94.

${ }^{20}$ Ibid., p. 134.

${ }^{21}$ Jeanneret, Ozenfant. Op. cit., p. 384.
} 
demuestran que la arquitectura tradicional contenía gamas más amplias de tonos en NCS, con más gamas amarillentas, próximas a los tonos NCS Y30R ó Y40R, y en general con menos negrura $\mathrm{NCS}^{22}$.

\subsection{Colores ausentes}

Es interesante detenerse a observar no sólo los colores seleccionados para Salubra I sino también los ausentes. Uno de ellos es el color negro, que se sustituye por otros colores bastante oscuros pero que en realidad pertenecen a otras familias de color-pigmento: marrón y siena. Sí que existen cuatro colores grises pero sólo dos de ellos pertenecen a los colores neutros N en NCS $(011,012)$. Estos cuatro colores grisáceos tienen distintos porcentajes de negrura, sin embargo el color más oscuro corresponde con un marrón (130) equivalente al tono NCS Y80R.

Resulta llamativa también la ausencia del blanco, siendo el color más parecido el 001, un tono amarillento de la familia NCS Y30R. El color blanco está ausente precisamente porque tiene un estatus excepcional. El blanco es previo a la policromía, es el color necesario para purificar la mirada hacia la forma arquitectónica, como señala Wigley $^{23}$. Le Corbusier considera que el blanco es fondo necesario para que el resto de colores asuman su significado. Así lo señala a propósito de la Villa Albert Jeanneret (París, 1923-1925): "La base será blanca, pero pintaremos dos de las paredes de rosa pálido (tierra siena tostado claro) y otra que se oponga, verde pálido; el resto blanco, el techo blanco" 24 . El color blanco es el del estuco o el de la pintura que se aplica directamente a los techos y a los fondos de las paredes, y no es necesario incluir un papel pintado de color blanco en la colección Salubra I. Volvemos a señalar que los cartones para seleccionar los colores de los claviers son de color blanco y no un gris medio como podría ser recomendable para evitar el fenómeno de los contrastes simultáneos a la hora de observar un color. Sobre el blanco es donde hay que visualizar la policromía arquitectónica, pero aún se puede ir más allá, pues Le Corbusier no sólo menciona el blanco como el fondo necesario sino también como el fin en sí mismo de toda la organización cromática. Así, señala Le Corbusier a propósito del Pabellón de l'Esprit Nouveau: "la cal brilla a consecuencia de la superficie de la pared que está en sombra (tierra sombra tostada o natural) a consecuencia de la pared que es cálida (ocres), a consecuencia de la pared que cede su protagonismo (sombras azuladas, etc.). Una casa completamente blanca parecería un pastel"25. En esta afirmación Le Corbusier parece querer trabajar al modo de los pintores impresionistas, quienes disponían acentos de color alrededor del blanco para destacar su luminosidad.

También llama la atención la ausencia de varias familias de color correspondientes a los amarillos verdosos y amarillos anaranjados y cuya nomenclatura correspondería con los números 06 y 07 en notación Salubra I. Efectivamente no existen colores entre los tonos NCS G40Y y Y60R, a excepción de cuatro colores sombra (060, 140, 141 y 142). Parece que Le Corbusier desplaza las gamas de amarillo cadmio hacia unos colores tierra, más bien oscuros y las gamas de amarillos verdosos hacia unos verdes escasamente amarillentos. Existe también

\footnotetext{
${ }^{22}$ García Codoñer, Ángela y otros: La arquitectura tradicional de Ontinyent: El color histórico. Valencia: Ajuntament d' Ontinyent, 2010.

${ }^{23}$ Wigley, Mark: White walls designer dressess: the fashioning of modern architecture. Cambridge: MIT press, 1995.

24 "La base sera le blanc; mais on peindra deux des murs en rose pâle (terre de Sienne brûlée claire), un autre qui fait vis-àvis, en vert pâle; le reste blanc, le plafond blanc." Le Corbusier, Polychromie Architecturale, Op. cit. p. 122.

25 'Le 'lait de chaux' étincelle à cause de ce pan de mur qui est sombre (terre d'ombre brûlée ou naturelle), de ce mur qui est chaud (ocres), de ce mur qui fuit (bleus, etc.). Entièrement blanche la maison serait un pot à crème." Le Corbusier: Almanach d'architecture moderne. Paris: Ed. Connivences, 1926. p. 146.
} 
una ausencia evidente en las familias de tonos ubicadas entre la NCS R20B y la R80B, es decir los violetas, que son descartados de su consideración arquitectónica Purista.

En 1951 Le Corbusier corrige estas ausencias de familias tonales en su nueva paleta de colores, los types couleurs, que empleó en las Unité d'Habitation o el Pabellón Suizo ${ }^{26}$. En esta paleta se representan todos los colores del espectro incluyendo un amarillo vivo, el violeta y el negro. Cuando ocho años después se publica la paleta de colores Salubra II encontramos algunos de estos colores ausentes en la primera versión: el color negro (4320E), un amarillo cadmio de tono NCS Y10R (4320W) y también un verde amarillento muy saturado NCS G80Y (4320F). En general se proponen colores con más porcentajes de negrura y cromaticidad NCS. Se abandonan los colores suaves por otros más llamativos.

Como señala Ruegg ${ }^{27}$ la arquitectura de Le Corbusier posterior a la II GM está dominada por una fuerte coloración, a veces en forma de "acentos cromáticos". El negro puro se dispone ahora cerca del blanco cuando al principio difícilmente aparecía como una superficie (exceptuando ciertos suelos fabricados con baldosas negras) y en general las texturas de los materiales empiezan a cobrar importancia. Sin duda una evolución evidente de la policromía arquitectónica que se hace menos rígida y más expresiva.

\section{Combinaciones de colores basadas en el contraste de tono NCS}

Un posible criterio de armonía reside en el empleo de colores que se encuentren en posiciones opuestas en el círculo cromático y que sean, por tanto, colores complementarios. Chevreul, Goethe, Munsell o Itten, estudiaron ya este principio de armonía que tuvo gran influencia en los pintores impresionistas y postimpresionistas como Signac o Seurat. Le Corbusier era conocedor sin duda de estas leyes de la complementariedad del color, pero sus cartas Salubra I no se construyen con un sistema ordenado de colores en el que se pueda observar la existencia de colores oponentes o complementarios siguiendo el criterio de Itten: rojo-verde, azul-naranja y amarillovioleta. Entre estos pares de complementarios, el amarillo-violeta ha de ser rechazado por no existir violetas en Salubra I. El naranja-azul podría ser algo significativo y el rojo-verde el mayor de ellos, pero en términos generales las combinaciones de Le Corbusier no parecen estar elegidas siguiendo una oposición estricta entre colores complementarios.

Tampoco se adecuan a los pares de colores oponentes que sostienen la construcción del espacio de color NCS: rojo-verde, azul-amarillo y negro-blanco. Si se proyectan sobre un plano bidimensional los colores y combinaciones Salubra I, se observa que pocas líneas pasan exactamente por el centro del círculo cromático NCS (fig. 2). Las relaciones entre los colores dibujan una figura con una geometría parecida a triángulos con una serie de vértices que corresponden con los colores principales de cada familia tonal, aquellos con más cromaticidad y negrura NCS.

Sin embargo, parece que existe un criterio de combinación que persigue un equilibrio entre colores cálidos y fríos y no un criterio estricto de complementariedad o de pares oponentes perceptivos. Heer ${ }^{28}$ asegura que azul y

\footnotetext{
${ }^{26}$ En la paleta de colores para la Maison du Pelerin en Ronchamp, se muestran dos colores amarillo cadmio, junto a un azul ultramar y un gris que nada tienen que ver con la escasa presencia de los amarillos en Salubra I. Este documento se encuentra en la Fundacion Le Corbusier (FLC Q1- 7. 118).

${ }^{27}$ Ruegg. Op. cit., pp. 66-83.

${ }^{28}$ Heer, Op. cit., p. 91.
} 
rojo fueron los pares básicos del sistema de color de Le Corbusier y que formaban un contraste que más allá de la pura óptica, respondía a cuestiones psicológicas, asociativas y arquitectónicas. Le Corbusier mismo señalaba que "La clasificación en dos grandes categorías de tonos fríos y cálidos aporta orden: cada color (tono y valor) se dirige bien hacia el lado luminoso (calidez, alegría, gozo, violencia) o hacia el lado sombrío (frescura, serenidad, melancolía, tristeza). [...] El rojo (y sus combinaciones de marrones, naranjas, etc...) fijan la pared, afirman su posición exacta, su dimensión, su presencia. Además, al azul se vinculan sensaciones subjetivas, de suavidad, calma, de agua natural, mar o cielo. Al rojo se asocian sensaciones de fuerza, de violencia. El azul actúa como un calmante del cuerpo, el rojo como un estimulante. Uno es descanso, el otro acción "29. Hay que señalar además que esta dualidad entre dos colores oponentes rojo-azul en términos de luz-sombra, calidezfrescura, alegría-serenidad, gozo-melancolía, violencia-tristeza, es una traslación literal de la teoría del color de Goethe $^{30}$ y sus parejas de oponentes amarillo-azul, a quienes asignaba las cualidades de distancia-cercanía, poder-blandura, repulsión-atracción y demás. Le Corbusier sustituye simplemente el amarillo del esquema de Goethe por el rojo.

El equilibrio entre colores fríos-cálidos explica bien los criterios de armonía para muchas de las combinaciones presentes en la colección Salubra I. En la figura 3 se ha señalado con un rayado el lugar geométrico de las combinaciones que manejan pares de colores opuestos frió-cálido y en ella se encuentran un gran número de muestras. Hemos considerado como colores fríos las familias de colores-pigmento del azul ultramar, azul cobalto, verde-amarillento y verde veronés; y como colores cálidos las familias del naranja, rojo bermellón y carmín. Los colores fríos pertenecen al rango entre NCS R80B y G40Y, y los colores cálidos al rango entre NCS Y30R y R10B. No incluimos ocres, sienas ni marrones entre los colores cálidos pues aunque pueden tener una proporción importante de rojo poseen poca cromaticidad NCS. Cualquiera de estos colores apagados combinado con un color frío destacará antes por su contraste de negrura o cromaticidad que por su tono. Los consideramos colores neutros en la oposición frío-cálido, junto con los grises.

En su estudio de las cartas Salubra I, Colli ${ }^{31}$ consideraba las gamas de la familia de color-pigmento verdeamarillento como colores cálidos, mientras que los carmines eran considerados colores fríos. Efectivamente el verde amarillento es un color más cálido que el verde veronés y eso explica que Le Corbusier prefiera combinar el verde amarillento junto al carmín, que es más frío, que no junto al rojo bermellón. Pero nosotros consideramos que tanto bermellón como carmín forman familias de color-pigmento cálidas, y que verde amarillento y verde veronés deben ser consideradas familias de colores fríos. Esto se debe a que la oposición de color frío-cálido en Le Corbusier es más conceptual que perceptiva. Si al rojo corresponde el papel de "fijar la pared", esta descripción se ajusta tanto para el carmín como para el bermellón y ambos deben ser considerados colores cálidos, a pesar del ligero contenido de azul en el caso del carmín. Examinando los colores verdes, tanto el verde amarillento como el verde veronés responden a la idea de "dar espacio" y deben ser considerados ambos colores fríos, a pesar de la ligera proporción de amarillo en el caso del verde-amarillento.

\footnotetext{
29 "La classification en deux grandes categories de tons chauds et tons froids apporte l'ordre: toute couleur (ton \& valeur) se dirige soit du côté lumière (chaleur, gaîté, joie, violence), soit du côté ombre (fraîcheur, sérénité, mélancolie, tristesse). [...] Le rouges (et ses composes bruns, oranges, etc. ...) fixe le mur, affirme sa situation exacte, sa dimension, sa présence. De plus, au bleu s'attachent des sensations subjectives de douceur, de calme, de paysage-eau, meu or ciel. Aur rouge s'attachent des sensations de force, de violence. Le bleu agit sur l'organisme comme un calmant, le rouge comme un excitant. l'un est au repos, l'autre est action". Le Corbusier. Polychromie architecturale. Op. cit., pp. 98, 115.

${ }^{30}$ Goethe. Op. cit. p. 158.

${ }^{31}$ Colli, Luisa Martina: "Le Corbusier e il colore: i Claviers Salubra”. En Storia Dell'arte. № 43. s.1.: s.e., 1981. p. $272-285$.
} 
4. Los cuadros representan cada uno de los claviers de Salubra I (1931). En abscisas se muestran en orden los color tons con su
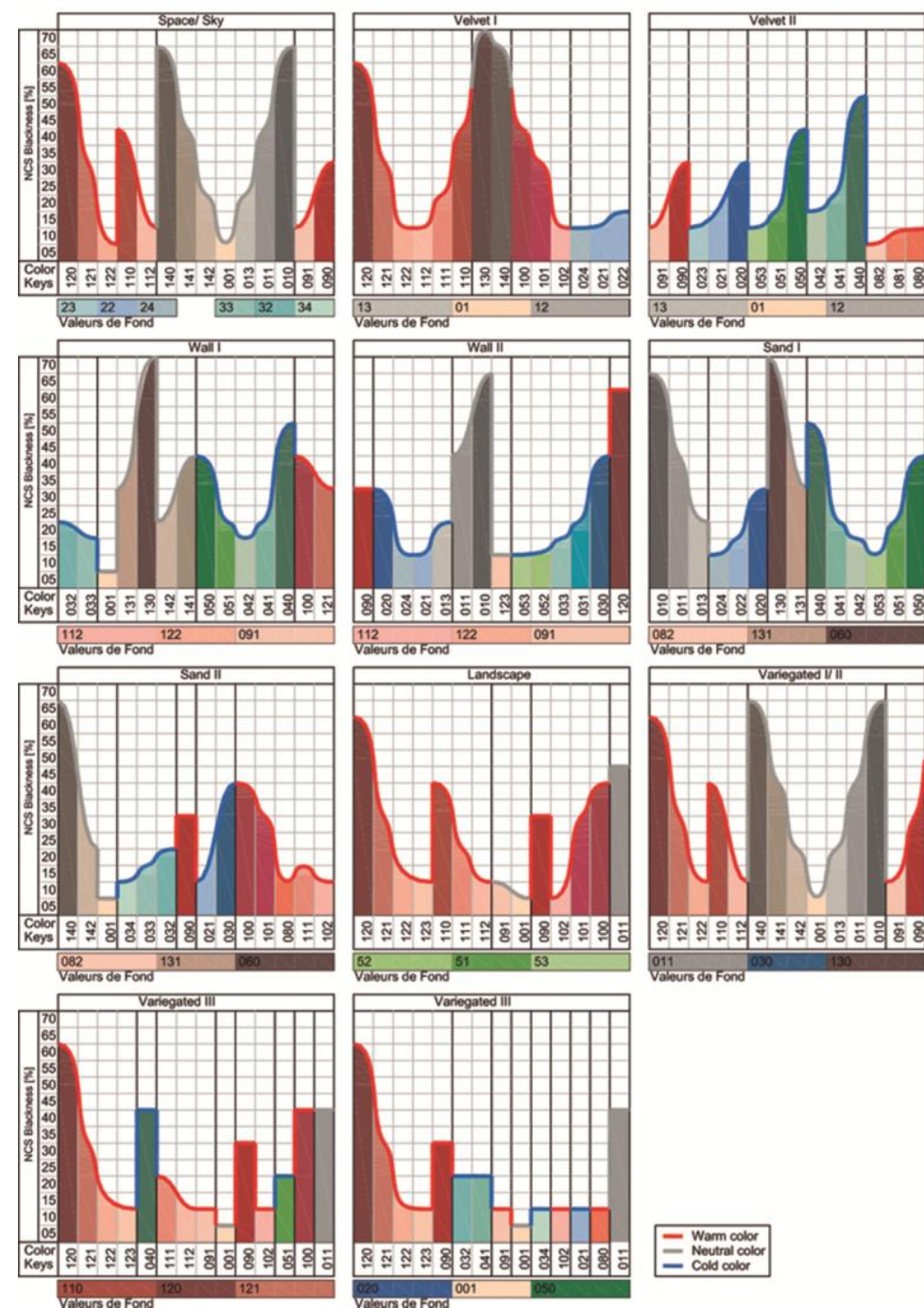

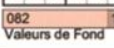
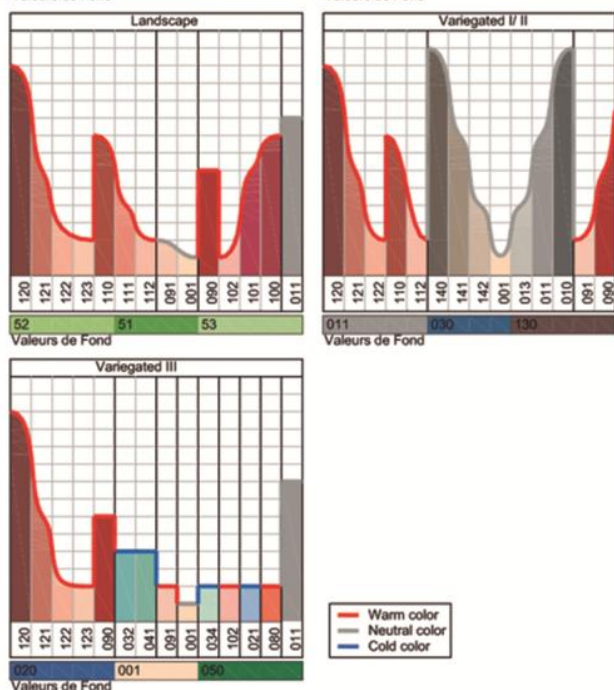

identificación Salubra I, así como los valeurs de fond en fila aparte. En ordenadas se indica el porcentaje de negrura NCS. Una arista roja, azul o gris indica si el color en cuestión es cálido, frío o neutro, respectivamente. Aristas negras verticales indican contrastes frío/cálido/neutro entre colores adyacentes. Se evidencian las secuencias y discontinuidades en la ordenación de los colores por

Le Corbusier. This work is licensed under a Creative Commons 4.0 International License (CC BY-NC-ND 4.0)

En la figura 4 se representan los color tons de los 12 claviers, indicando en la secuencia de cada uno de ellos el valor de negrura NCS de cada color y su pertenencia a los tonos fríos, cálidos o neutros. La finalidad es observar en ella los puntos de inflexión en los valores de negrura, así como las transiciones entre colores fríos y cálidos. Como si de una especie de línea de armonía se tratara podemos ver la presencia de pautas, simetrías o saltos, sin olvidar los valeurs de fond, que dan idea del timbre general de la composición. Vemos que el color rojo bermellón (090) sirve a menudo para resolver la transición entre gamas conflictivas o que no son preferidas: se interpone entre los azules cobalto y azules ultramar en Sable II, así como entre los sienas y carmines en Paysage. También en Bigarrée III se interpone entre los azules cobalto y los sienas. Llama la atención la escasa presencia de verdes y azules en claviers como Bigarrée III, así como entre los color tons de aquellos claviers donde los valeurs de fond son fríos (Espace, Ciel y Bigarrées I, II, III). 


\section{Conclusiones}

Hemos realizamos un nuevo estudio de las combinaciones de colores seleccionados por Le Corbusier en los claviers para la empresa de papeles pintados Salubra I en 1931, mediante su análisis en el espacio de color Natural Color System NCS, que permite comprender las variables perceptivas de los colores (tono, negrura y cromaticidad) y sus criterios de armonía.

Centrándose en el estudio de los tonos hemos observado que los 43 colores se deducen a partir de 12 colores principales a los que se añade blanco en distinta proporción. Al margen los 9 colores grises y neutros, la mitad de los restantes pertenecen a tonos fríos (NCS R80B a G40Y) y la mitad a tonos cálidos (NCS Y30R a R10B). Destaca la diferencia de estos colores amarronados y poco saturados con los propios de la arquitectura tradicional, a pesar del deseo de Le Corbusier de vincularlos con los "colores eternos".

Para completar el círculo cromático faltan colores en la gama de los amarillos (G40Y a Y60R) y los violetas (R10B a R80B). Tampoco aparecen el blanco, que se presupone para la arquitectura, ni el negro. Algunas de estas ausencias se suplen en las cartas Salubra II de 1959 y en edificios construidos después de la II GM.

Con respecto a los criterios de armonía se demuestra que las armonías de colores basadas en la similitud de tono son habituales, sobre todo si se consideran las parejas de dos color tons, y son especialmente significativas para las familias tonales del naranja, verde veronés e incluso carmín, donde parece ser un criterio de armonía preferido frente a otros. También existen gran cantidad de parejas de colores cuya armonía se basa en el contraste de tono, pero no tanto de colores complementarios y opuestos en el círculo cromático, sino de colores fríos y cálidos. La oposición entre colores fríos-cálidos fue señalada por el propio Le Corbusier y explica muchas de las combinaciones presentes en la colección Salubra I de 1931. Azul y rojo son considerados antónimos a nivel visual, psicológico, simbólico y arquitectónico.

En un estudio comparado del contraste frío-cálido y del claroscuro, se demuestra la presencia de ritmos y pautas en los claviers, habitualmente porque a un color principal suelen acompañarle otros colores de la misma familia tonal. Además se observa que el rojo bermellón sirve para resolver la transición entre gamas conflictivas o que no son preferidas.

\section{Agradecimientos}

Los autores agradecen especialmente la ayuda de la catedrática Ángela García Codoñer, directora del Grupo de Investigación del color de la UPV, por su profundo conocimiento del color. También la ayuda del catedrático José Herráez Boquera en el manejo de valores numéricos y su posterior dibujado en CAD; la ayuda del Dr. Pedro Cabezos Bernal en el modelado 3D en formato .pdf y la ayuda de Isabelle Godineau en la localización de documentos en la Fundación Le Corbusier. Además, este trabajo ha sido posible gracias a la financiación del Vicerrectorado de Investigación de la Universitat Politècnica de València (UPV, PAID-06-2012).

\section{Bibliografía/ Referencias}

Caivano, José Luis: La compleja relación con el color de uno de los maestros de la arquitectura moderna: Nueva edición de la Policromía Arquitectónica de Le Corbusier”. En Luminotecnia. № 84. Mar de la Plata (Argentina): Editores SRL, 2007. pp. 58-61. 
Chevreul, Michel Eugène: De la loi du contraste simultané des couleurs et de l'assortiment des objets colorés. Paris: Gauthier-Villars et fills; 1839. p 750. Traducción inglesa: Martel, C: The principles of harmony and contrast of colors and their applications to the arts. London: Longman, Brown, Green and Longmans, 1855.

Colli, Luisa Martina: "Le Corbusier e il colore: i Claviers Salubra”. En Storia Dell'arte. No 43. s.l.: s.e., 1981. p. 272-285.

En Lucan, Jaques (Ed.): Le Corbusier, une encyclopédie. Paris: Centre de Crêation Industrielle, 1987. pp. $97-$ 100.

Colli, Luisa Martina: "Hacia una policromía arquitectónica”. En Lucan, Jaques (Ed.): Le Corbusier, une encyclopédie. Paris: Centre de Crêation Industrielle, 1987. pp. 97-100.

Dempsey, Amy: Estilos, escuelas y movimientos: guía enciclopédica del arte moderno. Barcelona: Blume, 2002.

García Codoñer, Ángela y otros: La arquitectura tradicional de Ontinyent: El color histórico. Valencia: Ajuntament d' Ontinyent, 2010.

Goethe, Johann Wolfgang: Materialien zur Geschichte der Farbenlehre, Zur Farbenlehre. Tubingen: Cotta, 1810. Traducción inglesa: Eastlake, C.: Goethe's theory of colours. London: J. Murray, 1840.

Heer, Jean de: The architectonic colour: polychromy in the Purist architecture of Le Corbusier. Rotterdam: 010 Publishers, 2009.

Itten, Johannes: Kunst der Farbe. Ravensburg: Otto Maier Verlag, 1961. Versión inglesa por: Hagen, E. The elements of color. NY: Van Nostrand Reinhold, 1970.

Jeanneret, Ozenfant: “Le Purisme”. En: L’Esprit Nouveau. No 4. Paris: Ed. de L’Esprit Nouveau, 1920. pp 382 ff.

Jeanneret, Ozenfant: “Nature et creation”. En: L’Esprit Nouveau. N 19. Paris: Ed. de L'Esprit Nouveau, 1923. s.p.

Le Corbusier: "Polychromie architecturale". En Rüegg, Arthur (Ed.): Polychromie architecturale: Le corbusier's color keyboards from 1931 and 1959. Basel: Birkhäuser, 1997. pp 94-143.

Le Corbusier: Almanach d'architecture moderne. Paris: Ed. Connivences, 1926. p. 146.

Munsell, Albert: A grammar of colour. Mittineague, Massachusetts: The Strathmore paper company, 1921.

O’Connor, Zenna: “Colour Harmony Revisited”. En: Color Research and Application. N ${ }^{\circ} 35$ (4). Wiley Periodicals, 2009. pp 268-273.

Rüegg, Arthur: "Le Corbusier's Polychromie architecturale and his Color keyboards from 1931 and 1959". En Rüegg, Arthur (Ed.): Polychromie architecturale: Le Corbusier's color keyboards from 1931 and 1959. Basel: Birkhäuser, 1997. pp. 12-93.

Serra Lluch, Juan y otros: "Color combination criteria in Le Corbusier's purist architecture based on Salubra claviers from 1931”. En: Color Research and Application. Enero 2015 (Early view). Wiley Periodicals, 2015. DOI: $10.1002 / \mathrm{col} .21940$

Wigley, Mark: White walls designer dressess: the fashioning of modern architecture. Cambridge: MIT press, 1995. 